\title{
Study on the Design of Luxury Exhibition Space Based on Narrative Theory
}

\author{
Liu Ke ${ }^{1 \mathrm{a}}$, Liu Xiaodong ${ }^{2 b}$ \\ ${ }^{1}$ College of Fashion and Art Design, Donghua University, Yan 'an West Road, Changning District, Shanghai, China \\ ${ }^{2}$ College of Fashion and Art Design, Donghua University, Yan 'an West Road, Changning District, Shanghai, China
}

\begin{abstract}
This thesis will take the narrative theory as the foundation, analyzing the narrative method of luxury display space. The author adopts the literature research method, systematic analysis method, and interdisciplinary research method to study the luxury display space. By discussing the relationship between the narrative theory and luxury exhibition space, and combining with the cases of luxury brand exhibition space, this paper explores how to apply the narrative theory to the design of luxury exhibition space. Re-examining the spatial narrative from an interdisciplinary perspective is not only conducive to the emotional communication between brands and consumers, but also conducive to the value construction and promotion of luxury brands.
\end{abstract}

\section{Overview and Development of Narrative Theory}

Narrative means narrative a story, is one of the most accessible forms of human expression. The word narrative has both nominal and verbal meanings. As a noun, it means the story itself, while as a verb it refers to relate a story. The concept of narration was first used in literary works, and Long Diyong considers that the scope of narration is not confined to the narrow and excessive novel field ${ }^{1}$. In fact, a painting, a film and even a look are all kinds of narration.

The theory of narrative can be traced back to the ancient Greek period. Aristotle the word narrative in Poetics and illustrated its meaning. The appearance of narrative as a professional word was firstly proposed by The French critic TzvetanTodorov. He first used the term narrative in his Decameron, published in 1969, and emphasized that narratology as "the science of narrative works." ${ }^{2}$ Later, narratology gradually developed into "classical narratology" and "post-classical narratology".

For a long time, the narratology theory has been mainly used in the time dimension, and lacking discussion and research on the spatial dimension. However, in real life, time and space dimensions are inseparable. With the rise of post-modern culture, the theory of narrative has gradually matured. Since the 1980 s, scholars began to pay attention to the study of the spatial dimension of narratology. Narratology researchers admit that "space is not an external aspect of narrative, but an internal force that determines the development of narrative." ${ }^{3}$ The study of narratology of spatial dimension infuses space with cultural elements, lays emphasis on the mutual communication between space and people, and allows space to "speak".

\section{Concept and Current Situation of Luxury Display Space}

The interpretation of luxury good in the Oxford Advanced English-Chinese Dictionary is "a thing that is expensive and enjoyable but not essential". Wolfgang Latzler, Germany, in Luxury Brings Abundance, has made a relatively extensive broad interpretation of luxury good, which is not limited to the material product, but also includes aspects such as social interaction, tourism, leisure, etc., emphasizing that the surface of luxury good refers to the consumption of some products, but the deeper level more represents a way of life 4 .

According to the 2019 McKinsey China Luxury Report, although our country is under the new normal economic slowdown, the luxury goods market still shows no signs of weakness. In 2018, Chinese consumption of luxury goods at home and abroad reached 770 billion yuan on luxury goods, accounting for one-third of the global total. On average, every family spends nearly 80,000 yuan on luxury goods. Nevertheless, emerging Chinese luxury consumers lack awareness of brand history, culture and heritage, and they are more influenced by modern brand stories ${ }^{[5]}$. Therefore, the study of the narratology theory of luxury display space will provide some enlightenment for domestic brands to get involved in the luxury field.

Luxury exhibition space is not only a place to display goods, but also a container to convey brand culture and spirit to the audience. Moreover, it is also a space for luxury brands to have a dialogue with the audience. The exhibition space creates the entire environment through spatial planning, scenes layout, lighting management and other methods. Thus, the abstract brand concept and 
culture is presented to the audience in a concrete visual experience, which is also a way of effective communication between brands and consumers. Each luxury exhibition space conveys information and tells a story to us, in which each exhibit plays a different role, allowing viewers to experience different charm ${ }^{6}$. To put it simply, in the process of telling a story, luxury display spaces use their own characteristics and the environment of the entire space to help designers better interpret brand culture, product characteristics and their understanding of design and life.

The presentation will take different forms depending on the purpose and space. At present, the luxury display space basically displays the brand image through the showcase, store spaces, show fields and other forms. In the process of designing luxury exhibition space, quite a few designers have inadvertently used some narrative techniques, to allow consumers to have more involved with luxury brands, understand brand culture and experience brand stories according to the order of time during browsing. However, these narrative techniques are mostly pure time-linear methods, while the narrate theory has more diversified applications in luxury exhibition space.

\section{Research on Narrative Method in Luxury Display Space}

Currently, narratology theories have been one of the commonly used methods in luxury display design. By analyzing the different narrative methods in different types of luxury display space, the author further innovates the application of narrating in the luxury display space, so as to make the display space become a kind of story-telling material, which is also the development trend of luxury display space design in the future. The following will analyze the narrative method from three aspects: brand display space, thematic display space and cultural display space.

\subsection{Brand Display Space}

In the luxury display space, part of the display space is mainly to show the purpose of showing the development history, brand story and brand event of the luxury brand to the visitors. Its main purpose is to make consumers feel the cultural charm of the brand through spiritual communication, to gain brand recognition and drive related consumption. In this kind of exhibition space, narrative physical time axis can be put in. Whether it is a linear narrative mode or an incomplete linear narrative mode, the spatial sequence is arranged with time to deduce the spatial plot.

\subsubsection{Linear Model}

Gerard Genette, a French scholar, pointed out in Narrative Discourse that only through narration can narrative behavior be carried out and stories belonging to narrative content be presented ${ }^{7}$. It emphasizes the importance of chronology in arranging events in narrative. While the linear model in narrative space means that the plot of the whole space is arranged according to the time sequence in the narrative process, so that they are on a linear path, and thus designed as a space with clear order and distinct hierarchy. According to the order of time, the spatial narration of this linear model can be roughly divided into three forms: sequential narration, flashbacks, interposed narration.

It is one of the most common narrative methods to tell a story according to its development process. It enables visitors to visit in chronological order, to experience scenes at different time stages in the exhibition space, which is also in line with people's regular visiting habits. In the design of luxury exhibition space, this narrative method is often used in luxury historical topics, brand stories and other aspects. As an example, the 125-year-old Italian Classic design art exhibition of Bulgari is divided into seven chapters in chronological order. With exquisite jewelry, watches and works of art, it tells the legendary story of this world-renowned jewelry brand for the audience.

Although the sequential narration is clear and can also increase the sense of spatial hierarchy, sometimes lacking interest and no focus. While flashbacks can solve this problem well. Space flashback is to subvert the normal order and put important narrative plots in the front to draw suspense or motivate the viewer's interest in the tour ${ }^{8}$. In the exhibition design, an attractive object or plot is set at the entrance of the exhibition hall to increase the vividness of narration and arouse the interest of visitors.

And interposed narration stories refer to "inserting a heterogeneous event scene into a series of homogeneous event scenes to activate the original spatial system and create the contingency effect of a plot experience" ${ }^{19}$. In the design of luxury display space, insert the introduction of key techniques and important links in the brand growth and so on, which can enrich the narrative or emphasize the details. For instance, in Louis Vuitton's "Time-books" exhibition, scenes from the former studios of Asnieres were recreated on site. Meanwhile, craftsmen from France were also arranged to demonstrate how to make traditional leather. With the help of craftsman, visitors could participate in the making process and feel the beauty of French craftsmanship. This is of great benefit to the promotion of the whole space narrative plot and the emphasis of technology.

\subsubsection{Incomplete Linear Model}

Linear model of narrative space can clearly convey the narrative process and the plot of whole spaces, and it also makes the audience easy to understand a story or history. But most luxury display spaces begin to transition from linear model to incomplete linear model, appropriately weakening the straightforward narrative of the space, and adding more narrative links to enrich the whole narrative space. "We can no longer count on a time-ordered storyline, a history that is always in the process of accumulation, moving forward in terms of plot and outcome, so much happens that is incompatible with 
time." Western contemporary novelists are aware of the crisis of this narrative, so they introduce "simultaneity" into the creation of novels. This new narrative mode presents obvious spatial narration.

The incomplete linear model in the display space refers to the simultaneous development of events or plots under the guidance of a timeline, or the juxtaposition or cross narration of multiple different sequences ${ }^{10}$. Generally, this kind of incomplete linear narrative model presents a dendritic pattern, which is more layered and richer than the complete linear model, enabling the viewer to have a more comprehensive understanding of the whole story and relevant background.

"Christian Dior, Designer of Dreams" retrospective interprets the brand's artistic creation story lasting for more than 70 years from the perspective of China, presenting a feast of light and shadow of art and fashion. The retrospective is divided into themes and narrated in multiple chronological order. In addition, it invited eight famous Chinese artists to create art works and installations around the classical elements of Dior. While having a taste of Dior fashion art, you can also follow these avant-garde artists to learn about Dior's indissoluble relationship with China.

This fashion show is presented to the audience with a non-linear narrative method. In addition to the collocation of multiple timelines, it is also presented in different scenes and themes. Besides, photography, historical images, design sketches and other narrative techniques are also used to create a richer spatial experience, presenting a legendary dream journey for the viewer.

\subsection{Thematic Display Space}

\subsubsection{Single Thematic Narrative Mode}

The narrative method of thematic space is also very common in luxury display design. The theme is to collect all the contents or ideas to be expressed together, so that the sub-narrative is unified in this theme ${ }^{11}$, and the theme becomes the link throughout all the spaces so that the viewer can have a more intuitive understanding of the core content of the entire display space. Besides, the designer through a variety of themes to arrange luxury display space, can make the entire display space more colorful. It not only attracts viewers to have a deep understanding of luxury goods, but also strengthens their spiritual communication with designers.

For example, Mademoiselle Prive's "Into Chanel" exhibition in Shanghai's West Bund Art Center last year took the theme of "31 Cambon Street", "Glass Flower Field" and "18 Fontaine Square", three symbolic locations, telling the story of garment customization, perfume No. 5 and extreme jewelry, respectively. This exhibition will lead Chanel to the audience across time and space, analyze the creativity and inspiration of the brand development in the past hundred years for them, and let them truly enter the world of Chanel.

Therefore, the eternal principle of thematic spatial narrative design is theme first. In the design of luxury exhibition space, no matter the decoration of the space environment, the rendering of atmosphere and the arrangement of the plot, the purpose is to highlight its theme. The theme can greatly attract viewers, make them approach the exhibition automatically, unconsciously accept the information they see and feel, and subtly reduce the distance between them and luxury goods. Maybe this is the charm of luxury display design under the narratology theory.

\subsubsection{Comprehensive Narrative Mode}

Comprehensive spatial narration is the result of the comprehensive application of thematic narration and time narrations. Because in the design process of luxury display space, in order to meet the demand of rich space design plot, the display space may have clear themes while narrating with time or carry out theme parallel narration under sub-narrative space.

The "Spirit of Hermes" cultural exhibition travels around the world under the theme "Harness to its roots", which focuses on horse harness and its production, and takes us chronology through the history of the saddle and harness workshop since the inception of Thierry Hermès in 1837. Instead of chronology, the curators divided the subjects to show their relevance through the precise and open selection and display of exhibits. This is the way to combine the narrative of time with the narrative of thematic space. In this way, it integrates the wonderful story and craftsmanship, enhances the sensory experience of "the original intention of things", and sublimates the harmonious rhythm of Hermes and quality of life. At the same time, it also enables visitors to have a better understanding of the craftsmanship and the culture of Hermes, a luxury brand.

Various narrative modes of luxury display space design are not only the purpose of space narrative design, but also a way of design thinking. Each space uses different narrative techniques to convey information to viewers and tell them their own stories and cultures. It should not only give the audience a visual feast, but also have different levels of psychological experience, so as to deepen the audience's impression and imagination of luxury.

\subsection{Cultural Exhibition Space}

In comparison with other exhibition spaces, the biggest difference of luxury exhibition space with culture as the theme is that it pays more attention to the cultural deposits contained in the exhibition itself, which is mainly to spread culture and ideas. As a place to carry culture, transmit culture and continue memory, it is bound to have a certain influence on the psychological world of viewers, so as to promote them to better understand and remember this cultural memory. The famous Finnish architect Alvar Aalto once said, "The newest issue of modern design is to make rational methods go beyond the technical categories and into the human and psychological fields." 12 Therefore, the luxury display space in this situation is more suitable to use the 
narrative technique of summoning under psychological time.

The calling narrative technique in psychological time is to first use audio-visual context to create narrative plots, and subsequently use some narrative methods to mobilize the thinking and conscious activities in the audience's heart ${ }^{13}$. This makes the scene continue to extend in the viewer's mind, so as to form their own time in their hearts, and make the viewer have psychological feelings about the exhibition. In the calling narrative display space under psychological time, narrative space can be used as a blank space, abstraction, conceptualization and other techniques, so that the space itself can not complete the narrative. Only when viewers add their own thinking, imagination and understanding, can they complete the narrative together with the space.

Take the interactive exhibition "Decoding Hennessy" in 2018 as an example. It invites visitors to interpret the culture and stories behind cognac through a variety of interactive areas and a variety of thematic appreciation sessions. In the "Cognac Garden Walking area", through the interactive interpretation of the circular giant screen and sound, light and electricity, you will instantly pass through the French Cognac walking in the vineyard, personally experience the vineyard weather changes throughout the year. In "Cellar Secret," viewers can further explore the secrets behind the ancient brewing techniques and decode Hennessy's brewing process on an interactive wall. You can also have a dialogue with Hennessy's heirs travel through time and space, and you can share the unique charm of Hennessy.

This experiential interactive plot narrative plot makes the viewer through active experience understanding the theme of the exhibition in the exhibition hall and content. Moreover, they use technology to interact with the whole space, gradually understand the brand culture and slowly call out their inner feelings, then they can complete the enter narrative together with the space. This kind of exhibition enables the visitors to explore actively and go deep into their psychology so that the visitors can have a deeper cultural understanding and feeling.

\section{Example Verification of Luxury Display Space}

\subsection{Introduction to the Exhibition}

Since the birth of Louis Vuitton, the concept of "travel" has been rooted in the brand culture. In 2018, Louis Vuitton held an exhibition "Flight, Voyage, Travel" in Shanghai. The exhibition includes some designs from the brand's inception to its most recent, reflecting on the brand's great journey from its founding in 1854 to the present, as well as showing visitors the changes of The Times and vehicles over the past 100 years. From the time of the suitcase, antique bags, suitcases, into the public view, by them to tell us the legendary story of Louis Vuitton.

Designed by designer Robert Carsen, the exhibition consists of 15 chapters, interspersed with exhibits with Chinese roots. The exhibition opens with a hard case that is both iconic and symbolic, displaying Louis Vuitton's iconoclastic and energetic spirit of adventure. The following sections show visitors the craft, classic luggage, painting travel cases and other exhibits. According to the constant changes of transportation means, the way of travel is also changing constantly. From ship travel to train travel to air travel, Louis Vuitton tailors various kinds of bags and clothes to meet the travel needs according to different ways of travel and different needs of travelers. The exhibition ends with some iconic bespoke garments and craftsmanship of artisans.

This exhibition of Louis Vuitton once again lets more people appreciate the century-old brand's innovative spirit and superb craftsmanship, and this "culture of travel" is by no means a story that can be expressed in a few suitcases, it is the live track and artistic dedication of several generations.

\subsection{Research on the Comprehensive Narrative Method of Louis Vuitton}

Louis Vuitton's "Flight, Voyage and Travel" adopts a comprehensive narrative method. It divides the whole exhibition into 15 rooms according to the theme, and it presents the travel bags and related clothes designed for different users in each period in a certain chronological order. It allows the viewer to witness the microcosm of different times. In general, "travel" is a big theme, and several sub-narratives jointly narrated the history, culture and design of Louis Vuitton about travel, bringing us a wonderful journey.

As one of the constituent elements of narrative content, the plot helps to connect and sort out the spatial scattered points that are connect with each other. In this exhibition, there are also installations and plots that help the whole scene to be told together. As an example, in a train-themed exhibition hall, the window is designed in the form of a train carriage, with exhibits placed in the front and an LED screen playing moving images in the back. Being in the exhibition hall is like being on a moving train, giving people an immersive feeling. This form of exhibition increases the realism of narrative scenes, thus helping to narrate the space. Again, for instance, in the production area at the exit of the exhibition hall, a French craftsman teaches visitors how to make LV bags. Only when visitors have positive interaction with space through their consciousness and behavior can they deepen their memory and imagination of the brand. At this point, the narrative has moved to a deeper level, where it will remain in your memory and constantly evoke and re-narrate.

\section{Conclusion}

To sum up, the application of narrating theory to the design of luxury exhibition space can provide a more diversified and comprehensive research idea for the design. This will look at the design from a new perspective, so that the space design not only meets the practical value, but also can convey the spiritual culture. Through the display of various narrative modes, it brings 
a space for the viewer to speak, and enables the viewer to become a part of the narrative and blend into the whole narrative environment. It also conveys different spiritual qualities and life attitudes to us. In the context of personalized consumption today, the display space that can tell stories is one of the major trends in the design of luxury display space in the future, and the research on this aspect will be more in-depth.

\section{REFERENCES}

1. Long Diyong. Spatial Narratology: a New Field of Narrative Study [J]. Journal of Tianjin Normal University (Social Science Edition), 2008(6), 54.

2. Hua Xinxin. Spatial Narrative Research in Interior Design -- A Case Study of Youth Activity Space in Yangtze River Delta [D]. Nanjing Forestry University, 2017.

3. Guo Zengwei and Zhang Xiaoling. Space-time Narration in Byron's Long poem Don Juan [J]. Young Writer, 2015(35), 68-70.

4. Qiu Chenqing. Research on Louis Vuitton Brand Marketing in China [D]. Guangdong University of Foreign Studies, 2016.

5. Thomas Graziani. China Digital Luxury Report 2019, https://walkthechat.com/china-digital-luxury-report2018/

6. Wang Yingting. Discussion and Research on the Characteristics of Luxury Display Design [D]. East China Normal University, 2012.

7. Han Dong. Research on Multiple Narrative Modes of Luxury Brand Display Space [J]. Decoration, 2019,(311), 84-87.

8. $\mathrm{Mu}$ Yongbing. Research on Museum Space Design Based on the Theory of Spatial Narrates [D]. Lu Xun Academy of Fine Arts, 2019.

9. Lai Wanyi. Research on Landscape Cultural Expression Based on Spatial Narration -- A Case Study of Nantou Ancient City of Shenzhen [D]. Shenzhen University, 2018.

10. Kong Zhen. Research on Western Contemporary Spatialized Narrative Theory [D]. Shandong Normal University, 2011.

11. Wang Tiandan. Research on Exhibition Space Design Based on Spatial Narrative Theory -- A Case Study of Traditional Chinese Medicine Exhibition Center in Gion Hall [D]. Sichuan Academy of Fine Arts, 2018.

12. Xie Xuanhui. Narrative Research on Space Environment Design [D]. North China University of Technology, 2016.

13. Cheng Yanyan. Research on Narrative Space Design of Contemporary Cultural Architecture from the Perspective of Memory Theory [D]. South China University of Technology, 2018. 